\title{
Demography for Actuarial Students
}

\section{John A. Beekman}

\section{Introduction}

Demography is a very exciting subject. With a simple mathematical model, students can project a country's population forward $25,50,75$, even 100 years. They quickly see how small yearly growth rates can lead to staggering populations for future years. With a simple Markov chain, and powers of matrices, one can model rural-urban shifts in population, with its consequent reduction in births yet enlargement of social security needs.

University, college, and public libraries contain a wealth of population, mortality, and morbidity statistics which could be useful to both life insurance company and consulting actuaries. They provide bases for comparisons of mortality data, as a principal benefit. However, unless actuarial students are made aware of such data, they won't realize the existence of that wealth of statistics.

This paper will focus on six ideas from derography. They all can be useful to an actuary, yet none of them would be considered at any length if the actuary didn't study demography. They are:

(1) stable populations;

(2) population statistics and their uses;

(3) analytic expressions for $P(t)$, the population at time $t$;

(4) several probabilistic models in demography;

(5) population projections;

(6) finding probabilities from observed rates.

This list is not complete, but time and space limitations prohibit considering further ideas. 


\section{Stable Populations and Pension Applications}

Actuaries must possess a mastery of life tables, and at least part of pension mathematics. One of the more interesting concepts in both areas is that of a stable population. Such a group reflects growth, but is subject to fixed rates of fertility and mortality. The papers [5], [6] by Bowers, Hickman, and Nesbitt use general populations (including stable ones) to reflect population growth in pension plans. The very first component in the model pension plan, the population described in equation (1) on page 179 of [5], is apt to appear somewhat abstract to the reader who hasn't studied demography. This would not be the case for the person who had practiced with stable population mathematics. As is true of any mathematics subject, students learn best if they have problems to solve. The value of problem solving has been advocated by many, including the eminent mathematician Paul Halmos [10].

As one attempt to provide actuarial students with problems in demography, the author developed several exercise sets, described in ARCH 1980. 2, [2]. More recentiy, Professor Nathan Keyfitz and the author have been completing a book "Demography Through Problems" [12]. I deas from those sources will be used in this paper. The problems and solutions in [12] draw inspiration, ideas, and expressions from the theory and applications of demography expressed in Keyfitz's works [13] and [14].

For example 1, let us consider the following problem and its solution. Contrast the financial burden of a social security pension scheme in a country with a stationary population with the financial burden in a country with a stable population with growth rate of $r$. 
For the year $z$, we define

$R^{z}(x)=\frac{\text { Population over age } x}{\text { Population aged } 20 \text { to } x}$.

For a stationary population, with a retirement age of 65 ,

$R^{z}(65)=\frac{\int_{65}^{\omega} \ell(y) d y}{\int_{20}^{65} \ell(y) d y}$.

For a stationary population, one assumes a constant number $B$ of births each year.

In a stable population, one assumes that births follow an exponential function $B e^{r t}, t \geq 0$, where $r$ is the growth rate. This will affect the age distributions in future years. Thus, the function $\ell(y)$, should be replaced by $e^{-r y} \ell(y), 0 \leq x \leq \omega$, to reflect the smaller number of births y years ago.

$R_{r}^{z}(65)=\frac{\int_{65}^{\omega} e^{-r y} \mathcal{L}(y) d y}{\int_{20}^{65} e^{-r y} \mathcal{L}(y) d y}$

Let us now use part of the solution to Problem 38, Ch. III of [12].

$\ln R_{r}=\ln \left(\int_{65}^{\omega} e^{-r y} \ell(y) d y\right)-\ln \left(\int_{20}^{65} e^{-r y} \ell(y) d y\right)$

$\frac{d \ell n R_{r}}{d r}=\frac{-\int_{65}^{\omega} y e^{-r y} \ell(y) d y}{\int_{65}^{\omega} e^{-r y} \ell(y) d y}+\frac{\int_{20}^{65} y e^{-r y} \ell(y) d y}{\int_{20}^{65} e^{-r y} \ell(y) d y}$

$=m_{1}-m_{2}$ where $m_{1}=$ mean of the population 20 to $65, m_{2}=$ mean of the population over 65 . 
$\frac{d \ln R_{r}}{d r}=\frac{\frac{d}{d r} R_{r}}{R_{r}}=m_{1}-m_{2}$

$\frac{d}{d r} R_{r}=\frac{\Delta R_{r}}{\Delta r}$

Suppose that $m_{1}=35$, and $m_{2}=75$. Then $\frac{\Delta R_{r}}{R_{r}}=-40 \Delta r$.

Thus, for each percentage point of difference in $r$ there will be a difference of $40 \%$ of $R_{r}$ in the opposite direction. For example, $1 f$ one country is increasing at $2 \%$ per annum, and another country is stationary, the first country's burden is only $20 \%$ of the second country's burden.

It is true that the assumptions of a stable population may not persist with time. However, the actuarial student should be prepared to apply such ideas to his/her projects as the unfolding population may be better described by a stable population than by a stationary popuiation.

\section{Population Statistics and Their Uses}

There are a variety of ways for actuarial students to learn about ilfe tables. A thorough study of the underlying theory (e.g. Chapter 3 of [7]) is essential. A second step is an appreciation for the data from which life tables are constructed. Some sense of this can be grasped by studying the finished product, e.g. Life Table for the Total Population, United States, 1969-71, given on pages 14-17 of [7]. If the student has also learned exposed to risk techniques [1], and is assigned by his/her company to build one or more mortality tables, a better appreciation of data is gained. But few actuarial students have such opportunities in either summer or regular positions. This is where a study of demography can be very helpful. 
The United States Census Bureau has published a wealth of population and mortality statistics which can be found in many university, college, and public libraries. These can be used in several ways. For example, each student could be assigned a Standard Metropolitan Statistical Area (SMSA). For his/her SMSA, the student could do the following:

2. Create central death rates for the various age groups, by sex.

3. Compute crude death rates for 1965, and 1980 .

4. Compare the death rates for 1965 and 1980 due to malignant neoplasms versus major cardiovascular diseases.

5. Compute the infant mortality rates for 1965 and 1980 .

Students should be warned of misleading conclusions which could be reached by comparing crude death rates for two SMSA's. As explained in various references (including [21] and [22] two crude rates could be different even though the SMSA's had identical age-specific mortality rates, the difference occurring because of unlike distributions by age. It would also be possible for one SMSA to have a smaller crude death rate than that of another, even though its age-specific mortality rates were higher. This could be caused by the first SMSA having a younger population.

Users of life tables for specific geographic areas obtain valuable information by comparing such rates with those from a standard population, such as the state or possibly states surrounding a SMSA. To filter out the effects of the local population age distribution on the crude death rates, there are adjusted rates. The adjusted death rate by the direct method is

$$
\frac{\sum_{x} n^{p_{x}^{s}} \cdot n^{m} x}{\sum_{x} n^{p_{x}^{s}}}
$$


where ${ }_{n} P_{x}^{S}$ denotes the standard life table population between ages $x$ and $x+n$.

6. Calculate the adjusted death rate (direct method) for your SMSA.

IV. Analytic Expressions for $P(t)$, the Population at Time $t$ There are analytic expressions for populations changing with time, and these can be catalysts for data practice. The following problems reflect that.

7. Consider a logistic population model:

$$
P(t)=\left[A+B e^{-u t}\right]^{-1}, t \geq 0 .
$$

Verify that $P(t)$ satisfies the differential equation

$$
\frac{d P(t)}{d t}=u P(t)-u A[P(t)]^{2}
$$

8. Fit a logistic curve to the populations of Wisconsin from 1830 to 1980 as found in Census Bureau publications. Which volume or volumes did you use?

9. Fit a logistic population model to your SMSA. Let $P(0)=$ the population in 1960 , and use the fact that $P(\alpha)=1 / A=$ ultimate population. It would be helpful if you found the coordinates of the point of inflection.

A pioneering paper about the logistic population model was R. Pearl and L. J. Read, "On the Rate of Growth of the Population of the United States Since 1790 and Its Mathematical Representation", Proceedings, National Academy Sciences, 6 (1920), p. 275. The paper is discussed on pages 408-409 of [22]. Although the logistic projections for 1930, 1940, and 1950 were reasonably close to the eventual actual populations, there was a wide difference by 1960 . The same problem could occur in any logistic model. 
The following two problems and solutions utilize both the ideas of a stable population and census data.

10. A census ras been taken of a population assursed to be stable; the count at age $x$ is $C_{x}$, and that at age $y>x$ is $C_{y}$. Express the rate of increase in terms of $C_{x}, C_{y}$, and the survivorship function $\ell(x)$.

Solution: From stable population theory,

$$
\begin{aligned}
& c_{x}=b e^{-r x} \ell_{x} \\
& c_{y}=b e^{-r y} \ell_{y} .
\end{aligned}
$$

Taking logarithms reduces these to a pair of infar equations of the first degree whose solution in $\hat{r}$ is

$$
\hat{r}=\frac{1}{y-x} \ln \frac{c_{x} / l_{x}}{c_{y} / l_{y}}
$$

For this to be a good approximation we need $c_{x}$ to be the number of persons at age $x$ nearest birthday, and similarly for $c_{y}$. If $c_{x}$ and $c_{y}$ are the numbers at last birthday, a better approximation would be obtained by replacing $\ell_{x}$ and $\ell_{y}$ by

$$
L_{x} \fallingdotseq \frac{1}{2}\left(\ell_{x}+\ell_{x+1}\right) \text { and } L_{y} \fallingdotseq \frac{1}{2}\left(\ell_{y}+\ell_{y+1}\right) \text {. }
$$

11. In a population (that of MEXICO 1960) in which the census count at age 25-29 for females was $1,314,000$ and at age 5054 was 538,000 , and the number living in the life table for those two ages was 408,000 and 351,000 , find the rate of increase.

Solution. After adapting the previous formula to 5 -year ranges, we obtain $r=0.0297$. 
Many population projection models are also used in the mathematics of finance. The following example is illustrative of such problems.

12. The rate of increase of a population at time $t$ is $r(t)=$ $0.01+0.0001 t^{2}$. If the population totals $1,000,000$ at time zero, what is it at time 30 ?

\section{Solution}

$$
P(30)=1,000,000 \exp \left\{\int_{0}^{30}\left[0.01+0.0001 \mathrm{w}^{2}\right] \mathrm{dw}\right\}=3,320,117
$$

\section{Several Probabilistic Models in Demography}

Starting in 1983-84, many actuarial students will be learning actuarial mathematics from two excellent textbooks authored by Bowers, Gerber, Hickman, Jones, and Nesbitt [7]. As stated on page 5 of that work: "The sharpest break between the approach taken in these books and in earlier English language textbooks on actuarial mathematics is the use of a much fuller probabilistic approach in the treatment of mathematics of life contingencies." Some topics in demography lend themselves to reinforcing the need for a good knowledge of probability. Several of these topics will be pursued here.

13. Let $X_{1}, X_{2}, \ldots, X_{n}$ be independent random variables, each having distribution

$$
X=\left\{\begin{array}{l}
1 \text { with prob. } q_{x} \\
0 \text { with prob. } p_{x}
\end{array}\right.
$$

Define a random variable

$$
R=\sum_{i=1}^{n} X_{i}, E\left\{\sum_{i=1}^{n} X_{i}\right\} .
$$


(a) Find $E(R)$.

(b) Justify an expression for $\operatorname{Var}(R)$.

(c) Set up $95 \%$ confidence limits for $R$.

(d) How would you use these ideas in analyzing the mortality data for your SMSA? What stancard population might you have considered?

14. Assume that the probability of a girl on a particular birth is $p$, and of a boy is $q=1-p$. Let $N=$ raridom number of children for a couple with the family goal of having one girl. Finc $E(N)$.

15. Assume the same probabilities as in Problem 14. Let $N=$ rardom number of children for a couple whose family goal is to have one boy and one girl. Find $E(N)$.

16. Let $S=\sum_{i=1}^{5,000,000} X_{i}$ where each $X_{i}$ represents the random number of children to a newly married couple. Find $E(S)$ under family strategies one and two. By how much do they differ when $p=0.5$ and $q=0.5$ ?

Solution. Since the answers to problems 14 ard 15 were $E(N)=p^{-1}$, and $E(N)=q^{-1}+p^{-1}-1$, the values for $E(N)$ will be 2 and 3 when $p=q=.5$, and the values for $E(S)$ will differ by $5,000,000$ ! 


\section{Population Projections}

Actuaries who work for the Social Security Administration or the Bureau of the Census would make heavy use of the mathematics for population projections. To the extent that actuarial students do not know where their professional lives may take them, they should all have some exposure to those techniques. They are explained in Chapters 2, 3, and 4 of [13], Chapter 8 of [14], Chapters 11, 12, and 13 of [16], the paper [17], Chapters 12, 13, 14, and 15 of [19], Chapters 9 and 17 of [9], Chapter 8 of [18], Chapters 24 and 25 of [21], and Chapter 14 of [22]. These references will provide the reader with lengthy examples of population projections for Australia, Britain, France, United States, and many other countries. Most of the authors either are or have been members of the Institute of Actuaries, or the Society of Actuaries which increases the value of their books for actuarial students.

We will focus on some population projection techniques, which utilize probabilistic models.

It is possible to use a Markov chain to model rural-urban shifts in population, with its consequent reduction in births yet enlargement of social security needs. The following paragraphs are based on [3], and [4]. We consider a Markov chain with states of nature u(urban dweller) and r(rural dweller). Let the matrix of transition probabilities be:

$$
M=\left(\begin{array}{ll}
m_{u u} & m_{u r} \\
m_{r u} & m_{r r}
\end{array}\right)
$$


Assume that the initial distribution of urban and rural dwellers is:

$$
p(0)=\left(\begin{array}{l}
p_{u} \\
p_{r}
\end{array}\right) \text {. }
$$

The superscript. (0) on $P$ denotes the initial time. The distribution $t$ years later is given by

$$
p(t)=\tilde{M} t_{p}(0)
$$

where $\widetilde{M}$ is the transpose of $M$.

17. Assume that

$$
M=\left(\begin{array}{ll}
0.999 & 0.001 \\
0.020 & 0.980
\end{array}\right) \text {, }
$$

$P_{u}=8,000,000$, and $P_{r}=77,000,000$. Find $p(10)$.

The Markovian model can be improved to provide for growth. The population projections then become:

$$
\begin{aligned}
& p(1)=\tilde{M}_{e} r_{p}(0) \\
& p(2)=\widetilde{M} r_{p}(1)=\widetilde{M}^{2} e^{2 r_{p}(0)} \\
& \cdot \cdot \\
& p(10)=\tilde{M}^{10} e^{10 r_{p}(0)}
\end{aligned}
$$

18. Assuming that $r=0.02$, find $p(10)$.

The effects of rural-urban shifts on aggregate births can be learned from the Markovian model. If the overall birth rates in urban and rural parts are $b_{u}$ and $b_{-r}$, respectively, arranged in a horizontal vector $B$, then the births at time $t$. are 


$$
{ }_{B P}(t)=\left(\begin{array}{ll}
b_{u} & b_{r}
\end{array}\right)\left(\begin{array}{ll}
m_{u u} & m_{r u} \\
m_{u r} & m_{r r}
\end{array}\right)^{t}\left(\begin{array}{l}
e^{t r_{P_{u}}} \\
e^{t r_{P_{r}}}
\end{array}\right) \text {. }
$$

19. Assume that $b_{u}=0.035$ and $b_{r}=0.050$.

Compute $B e^{10 r_{P}}(0)-B \widetilde{M}^{10} e^{10 r_{P}} P(0)$ to see the difference in aggregate projected births for the eleventh year from the initial time point. A quick view of part of the solutions shows the point of these problems.

17. $p(10)=\left(\begin{array}{l}21,950,384 \\ 63,049,616\end{array}\right)$.

18. $p(10)=\left(\begin{array}{l}26,810,259 \\ 77,008,975\end{array}\right)$.

19. $B e^{10 r_{p}(0)}=5,044,393$

$$
\tilde{B M}^{10} \mathrm{e}^{10 r_{p}(0)}=4,788,808
$$

The difference is 255,585 .

The statistical estimation of the transition probabilities of a Markov chain can be based on the principle of maximum likelihood (see, e.g., pages 240-242 of Ross [20]). Such an estimator of the probability that the process goes from state $i$ to state $j$ is obtained from the proportion of time that the process, when leaving state $i$, next enters state $j$. Thus,

$$
\begin{aligned}
& \hat{\mathrm{m}}_{u r}=\frac{\text { Twelve Month Rural Increase--Natural Rural Increase }}{\text { July } 1 \text { Estimate of Urban Population }} \\
& \hat{\mathrm{m}}_{\text {ru }}=\frac{\text { Twelve Month Urban Increase--Natural Urban Increase }}{\text { July 1 Estimate of Rural Population }}
\end{aligned}
$$


Then properties of Markov chains would yield the estimates

$$
\hat{m}_{u u}=1-\hat{m}_{u r} \text {, and } \hat{m}_{r r}=1-\hat{m}_{r u}
$$

\section{Finding Probabilities from Observed Rates}

Life tables have been calculated for at least 300 years, and census and mortality data have been collected for a much longer period of time. Census data is usualiy found as of the middie of a calendar year, and mortality data is for a calendar year. Thus, one can obtain rates such as

$$
5^{M_{65}^{z}}=\frac{5^{D^{Z}} 65}{5^{L^{z}}}
$$

However, for many purposes one needs to know the probability $5^{9} 65$. There are a number of transformations of such rates to probabilities. The paper [15] by Keyfitz shows that

$$
\frac{\ell_{x}+5}{\ell_{x}}=\frac{1-2.5\left({ }_{5} M_{x}\right)}{1+2.5\left({ }_{5} M_{x}\right)}
$$

if one assumes that deaths are uniformly distributed through the five years.

The single problem which we shall examine is this section utflizes the ideas of a stable population, observed rates, and a life table.

20. Local stability exists when the population $p(x+t)=$ $e^{-r t} \ell(x+t), 0 \leq t \leq n$. Prove algebraically that under local stability

$$
n^{M} x+r=\frac{\ell_{x}-e^{-r n} \ell_{x}+n}{\int_{0}^{n} e^{-r t} \ell(x+t) d t}
$$


Explain in words that the two sides have to be equal because each represents the rate of flow through age groups.

Solution.

Under local stability,

$$
n^{M} x=\frac{\int_{0}^{n} e^{-r t} \ell(x+t) \mu(x+t) d t}{\int_{0}^{n} e^{-r t} \ell(x+t) d t} .
$$

Using the definition of $\mu(x+t)$ and integration by parts,

$$
\begin{aligned}
& \int_{0}^{n} e^{-r t} \ell(x+t)_{\mu}(x+t) d t=-\int_{0}^{n} e^{-r t} d \ell(x+t) \\
& =-e^{-r n} \ell_{x}+n+\ell_{x}-r \int_{0}^{n} e^{-r t} \ell(x+t) d t .
\end{aligned}
$$

A little algebra completes the proof.

The left side is the observed age-specific death rate for an individual between exact ages $x$ and $x+n$, plus the annual rate $r$ of increase in births. This total rate of flow in the observed population equals the flow with the life table functions and assumed birth function.

\section{Demography Papers for Actuaries}

Actuarial students who have studied demography have a marked advantage in understanding papers and books which are based (at least partially) on such knowledge. Two demography-based papers will now be briefly described.

Demography is the study of population dynamics. One tool for the 
analys is and projection of labor force mobility and participation is a working life table. Such a table is helpful for analyzing work-force progression, trends of retirement, and the money-value of an individual. The customary methods of constructing working life tables are based on a unimodal curve of labor force participation by age and a single lifetime entry and exit from the labor force. Jan M. Hoem has developed a new method of construction in [11]. It is based on the theory of Markov chains with a continuous time parameter. These are more appropriate models because they can reflect the dynamics of labor force participation. Data for labor force flows can be generated from periodic labor force surveys. There are several advantages to the new method. It accounts for multiple entries and exits which traditional methods do not do; and it allows for the bimodal curve of work rates by age for females. A major idea which is developed is wcrk-life expectancies by age ( $w i$ th and without mortality). Extensive data on the labor force mobility of Danish males, 1972-74, is anaiyzed, and illustrates the new method.

A second paper which illustrates effective use of demography in an actuarial setting is [8] by Robert L. Brown. It analyzes the effects of the changing Canadian demographic profile on the dependency ratios from 1976-2071, impact on health expenditures, projections for the Canada/ Quebec Pension Plan, future mortality rates, increased female labor force participation rates, and possible future retirement ages.

The increased female labor force participation rates have created a new market for insurance and annuities. This also explains the increased deriands for earlier and fuller vesting in private pensions. The Canada/ Quebec Pension Plan has special "drop-out' provisions so that the years which women spend out of the labor force as homemakers will not affect their benefits. It would appear that the Markov chain techniques which 
Jan Hoem has developed for working life tables would be helpful here.

The pension burden $T_{x}^{z} /\left(T_{20}^{z}-T_{x}^{z}\right)$ is tabulated for $x=65,60$, 55 , and $z=1976,2001,2031$. For a retirement age of 65 it has values $15.60 \%, 18.41 \%, 33.37 \%$ for the given years. These numbers as well as youth dependency ratios $\left(T_{0}^{z}-T_{18}^{z}\right) /\left(T_{18}^{z}-T_{65}^{z}\right)$ are portrayed in Figure 2. That figure also traces the tidal wave of births in the late 1950 's and the early 1960's through the age bracket 10-24 in 1976 to $35-49$ in 2001 through $65-79$ in 2031.

A thorough analysis is made of the impact on health expenditures of changes in the demographic profile. This included projections of per capita government-insured health expenditures based on low and high demographic growth from 1976-2001, as contrasted to per capita GNP growing at $3 \%$ or $2 \approx$ a year.

\section{Concluding Remarks}

In closing, it should be emphasized that the six ideas presented, the two demography-based papers which were described, and the list of references are not exhaustive by any means. There is a large wealth of concepts and population statistics that the actuarial student, and the practicing actuary should utilize. The author hopes that this paper will encourage greater utilization of those professional resources. 


\section{REFERENCES}

[1] Batten, Robert W., Mortality Table Construction, Prentice-Ha17, Englewood Cliffs, N. J., 1978.

[2] Beekman, John A., "Problems In and A Syllabus for Demography", Actuarial Research Clearing House, Issue 1980.2, 9-15, Society of Actuaries, Chicago.

[3] Beekman, John A., "Several Demographic Projection Techniques", Rural Demography, $\underset{2}{2}(1981), 1-11$.

[4」 Beekman, John A., "Population and Social Security Projections for Bangladesh", to appear in Proceedings of NATC Advanced

Study Institute, University of Leuven, Belgium, Reidel Publ., Holland, 1984.

[5] Bowers, Newton L., James C. Hickman, Cecil J. Nesbitt, "Introduction to the Dynamics of Pension Funding", Transactions, Society of Actuaries, 28 (1976), 177-203.

[6] Bowers, Newton L., James C. Hickman, Cecil J. Nesbitt, "The Dynamics of Pension Funding: Contribution Theory", Transactions, Society of Actuaries, 31 (1979), 93-119.

[7] Bowers, Newton L., Hans U. Gerber, James C. Hickman, Donald A. Jones, Cecil J. Nesbitt, Actuarial Mathematics, Volumes I and II, Society of Actuaries, Chicago, 1983, and 7984.

[8] Brown, Robert L., "Actuarial Aspects of the Changing Canadian Demographic Profile", Transactions, Society of Actuaries, $34(1982), 13-30$. 
[9] Cox, Peter R., Demography, 5th Ed., Cambridge University Press, Cambridge, 1976.

[10] Halmos, P. R., "The Heart of Mathematics", The American Mathematical Monthly, 87 (1980), 519-524.

[11] Hoem, Jan M., "A Markov Chain Model of Working Life Tables", Scandinavian Actuarial Journal, 1977, 1-20.

[12] Keyfitz, Nathan and John A. Beekman, Demography Through Problems, Springer-Verlag, New York, Heidelberg, 1984.

[13] Keyfitz, Nathan, Introduction to the Mathematics of Popuiation: With Revisions, Addison-Wesley, Reading, Mass., 1978.

[14] Keyfitz, Nathan, Applied Mathematical Demography, john V'i ley \& Sons, New York, 1977.

[15] Keyfitz, Nathan, "Finding Probabilities from Observed Rates or How to Make a Life Table", The American Statistician, February, $1970,28-33$.

[16] Keyfitz, Nathan, Population Change and Social Policy, Abt Books, Cambridge, Mass., 1982.

[17] Keyfitz, Nathan, "The Limits of Population Forecasting", Population and Development Review, $\underset{\sim}{\sim}$ (1981), 579-593.

[18] Pollard, A. H., Farhat Yusuf, G. N. Pollard, Demographic Techniques, 2nd Ed., Pergarmon Press, Sydney, 1981.

[19] Pressat, Roland, Demographic Analysis, Aldine-Atherton, Chicago, 1972 (English transiation of L Analyse Demographique, Presses Universitaires de France, Paris, 1969. 
[20] Ross, Sheldon M., Introduction to Probability Mode1s, Academic Press, New York, 1972.

[21] Shryock, Henry S., Jacob S. Siegel, The Methods and Materials of Demography, Vols. I and II, U.S. Government Printing Office, Washingtion, D.C., 1971

[22] Spiegelman, Mortimer, Introduction to Demography (Revised Edition), Harvard University Press, Cambridge, 1968. 